\title{
Observations and theoretical evaluations of color changes of traveling light beams caused by optical rotation phenomena in sugared water and their applications for educational purposes
}

Seika Tokumitsu, Makoto Hasegawa

Seika Tokumitsu, Makoto Hasegawa, "Observations and theoretical evaluations of color changes of traveling light beams caused by optical rotation phenomena in sugared water and their applications for educational purposes," Proc. SPIE 10452, 14th Conference on Education and Training in Optics and Photonics: ETOP 2017, 1045219 (16 August 2017); doi: 10.1117/12.2266609

Event: 14th Conference on Education and Training in Optics and Photonics, ETOP 2017, 2017, Hangzhou, China 


\title{
Observations and theoretical evaluations of color changes of traveling light beams caused by optical rotation phenomena in sugared water and their applications for educational purposes
}

\author{
Seika Tokumitsu, Makoto Hasegawa* \\ Chitose Institute of Science and Technology, 758-65 Bibi, Chitose, Hokkaido, JAPAN 066-8655
}

\begin{abstract}
Investigations were conducted for the purposes of understanding coloring phenomena to be caused by optical rotation of polarized light beams in sugared water and realizing their applications as educational tools. By allowing polarized laser beams in red, blue or green to travel in sugared water of certain concentrations, changes in their intensities were measured while changing a distance between a pair of polarizing plates in the sugared water. An equation was established for a theoretical value for the angle of rotation for light of any colors (wavelengths) travelling in sugared water of any concentrations. The predicted results exhibited satisfactory matching with the measured values. In addition, the intensities of transmitted laser beams, as well as colors to be observable when a white-color LED torch was employed as a light source, were also become predictable, and the predicted results were well-matched with the observation results.
\end{abstract}

Keywords: Polarization, optical rotation, angle of rotation, sugared water, science education, physics education

\section{INTRODUCTION}

Polarization is one of suitable phenomena that can attract people's attentions at science demonstration events aiming to stimulating and enhancing their interests in various fields of sciences and technologies. It can be also served as an effective subject for allowing undergraduate and graduate students to study and understand the nature of light as waves. For example, by employing a pair of polarizing plates and a piece of certain plastic material disposed between those polarizing plates, various beautiful colors can be observed. As another scheme, optical rotation phenomena of polarized light beams in sugared water can also exhibit certain colors ${ }^{1}$.

One problem of the coloring phenomena to be caused by optical rotation of polarized light beams in sugared water is difficulties in explanations of the phenomena as well as theoretical predictions of colors to be observed. More specifically, in the authors' brief surveys, any theoretical and mathematical explanations regarding predictions of colors to be observed in the phenomena cannot be found. Thus, in this paper, some investigations have been conducted for the purposes of understanding the coloring phenomena caused by optical rotation of polarized light beams in sugared water and realizing their applications as educational tools.

First, by allowing polarized laser beams in either of red, blue, or green colors to travel in sugared water of certain weight concentrations, changes in their spectroscopic intensities were measured while changing a distance between a pair of polarizing plates disposed in the sugared water. As a result, the intensities of the transmitted light beams were found to change in a regular manner with certain intervals in accordance with the distance between the polarizing plates. The intervals were different dependently on their colors and the concentrations of the sugared water. Then, the angle of rotation for each color was determined based on the measured results.

Thereafter, the authors tried to obtain theoretically expectable values for the angle of rotation for any colors (wavelengths). Although any suitable theoretical relationships could not be found in the literatures, the authors successfully established an equation that can provide us with a theoretically expectable value for the angle of rotation for light beams of any colors (wavelengths) travelling in sugared water of any concentrations. The theoretically expected results were then confirmed to show satisfactory matches with the measured results. Based on the theoretical results obtainable from the equation, the intensities of transmitted laser beams, as well as colors to be observable when a whitecolor LED torch was employed as a light source, were also become predictable, and they were well-matched with the actual observation results.

*hasegawa@photon.chitose.ac.jp; phone+81-123-27-6059; fax +81-123-27-6059

14th Conference on Education and Training in Optics and Photonics: ETOP 2017, edited by Xu Liu,

Xi-Cheng Zhang, Proc. of SPIE Vol. 10452, 1045219 • ( ) 2017 ICO, IEEE, OSA, SPIE

CCC code: $0277-786 X / 17 / \$ 18 \cdot$ doi: $10.1117 / 12.2266609$ 


\section{OPTICAL ROTATION OF POLARIZED LIGHT BEAMS}

When linearly polarized light beams are allowed to be incident on a certain type of materials, its polarization axis may be rotated. Such a phenomenon is called as optical rotation, and the material leading to the optical rotation is referred to as an optically active material. Optically active materials have chiral centers in their molecules. Among various optically active materials, a typical and easily available one is sucrose, which is a main component of sugar. Figure 1 shows the structure of a sucrose molecule, which includes many chiral atoms (indicated with *).

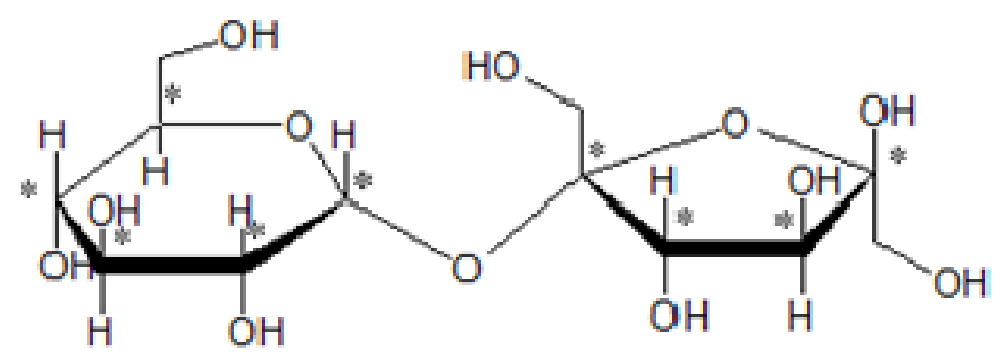

Figure 1. Molecular structure of sucrose.

For optically active materials, Maxwell's equations can be expressed as follows:

$$
\begin{gathered}
-\sqrt{\varepsilon_{0} \mu_{0} n}\left(\boldsymbol{k}_{\mathbf{0}} \times \boldsymbol{H}\right)=\boldsymbol{D}-i \frac{c}{g} \boldsymbol{H} \\
\sqrt{\varepsilon_{0} \mu_{0} n}\left(\boldsymbol{k}_{\mathbf{0}} \times \boldsymbol{E}\right)=\boldsymbol{B}-i \frac{c}{g} \boldsymbol{E}
\end{gathered}
$$

Among these equations, $g$ is called as a rotation factor, and expressed as follows:

$$
g \equiv \frac{2 \pi c}{\lambda_{0}} \cdot \frac{2+\frac{\varepsilon}{\varepsilon_{0}}}{3} N \beta
$$

where $\lambda_{0}$ is the wavelength of light in vacuum, $N$ is the number of molecules in the volume of $1 \mathrm{~cm}^{3}$, and $\beta$ is a constant unique to a material.

Assuming that linearly polarized light beams are travelling along the z-axis, the light beams can be in general expressed as follows:

$$
E=E_{0} \exp i\left\{\omega t-\left(\frac{2 \pi}{\lambda_{0}}\right) n z+\varphi_{0}\right\}
$$

Sugared water is a liquid, and thus can be considered as isotropic. When the light beams are allowed to travel in such an isotropic optically active material, the light beams can be considered as linearly polarized light beams which are obtained by combining (overlapping) right-handed circularly-polarized light beams and left-handed circularly-polarized light beams. For those combined light beams, equation (4) can be modified as follows:

$$
\boldsymbol{E}(z)=E_{0} \exp \left(-i \frac{\omega}{c} n_{0} z\right)\left(\boldsymbol{A}_{x} \cos \theta-\boldsymbol{A}_{y} \sin \theta\right)
$$

where

$$
\theta=\frac{\omega}{\mathrm{c}} \cdot \frac{\left(n_{-}-n_{+}\right)}{2} \mathrm{z}=\frac{2 \pi}{\lambda_{0}} g_{0} Z
$$

Specifically, when linearly polarized light beams are allowed to be incident onto an optically active material and travel therein, its polarization axis rotates by an angle $\theta$ over a travel distance $z$. For the travel distance of $z=1 \mathrm{~cm}$, the rotation angle is indicated as $\phi$, and expressed as follows: 


$$
\frac{\theta}{\mathrm{z}} \equiv \phi=\frac{2 \pi}{\lambda_{0}} g_{0}=\frac{4 \pi^{2} N c}{\lambda_{0}^{2}} \cdot \frac{2+\varepsilon / \varepsilon_{0}}{3} \beta
$$

The above angle $\phi \mathrm{rad} / \mathrm{cm}$ is called as the angle of rotation or the rotation power, indicating the rotation angle of linearly polarized light beams over the travel distance of $z=1 \mathrm{~cm}$ in an optically active material ${ }^{2}$.

When considering influences of dielectric dispersion, equation (7) can be re-written as follows:

$$
\phi=\frac{4 \pi^{2}}{3} N c \beta\left(\frac{2+\varepsilon_{\infty}+S_{j}}{\lambda^{2}}+\frac{S_{1} \lambda_{1}^{2}}{\lambda^{4}} \cdots\right)
$$

As can be seen, equation (8) includes $\lambda^{-4}$ or higher orders. However, in accordance with Biot's inverse square law, the specific angle of rotation $\alpha$ can be considered to be approximately proportional to $\lambda^{-2}$. Thus, in equation (8) for the angle of rotation $\phi$ in visible wavelength regions, $\lambda^{-4}$ or higher orders are assumed to be similarly ignorable. Then, equation (8) can be expressed as follows:

$$
\phi=\frac{4 \pi^{2}}{3} N c \beta\left(\frac{2+\varepsilon_{\infty}+S_{j}}{\lambda^{2}}\right)
$$

In the above explanation, the specific angle of rotation $\alpha$ is employed to express the angle of rotation for liquids. More specifically, the specific angle of rotation $\alpha$ indicates the angle of rotation in a liquid, determined as the angle of rotation in the case where light beams travel over $10 \mathrm{~cm}$ in a liquid containing an optically active material at the concentration of $C \mathrm{~g} / \mathrm{cm}^{3}$. By using the molecular weight $M$ of the optically active material contained in the liquid and the Avogadro's number $N_{A}$, the specific angle of rotation $\alpha$ can be expressed as follows:

$$
\alpha=\frac{\phi}{C} \cdot \frac{1800}{\pi}=\frac{g_{0}}{\lambda_{0}} \cdot \frac{3600}{C}=\frac{N_{A} \phi}{N \cdot M} \cdot \frac{1800}{\pi}\left[\mathrm{deg} / \mathrm{dm} \cdot \mathrm{g} \cdot \mathrm{cm}^{-3}\right]
$$

\section{INTRODUCTION OF THE GENERAL EQUATION FOR THE ANGLE OF ROTATION IN SUGARED WATER}

By re-writing equation (10), the following expression can be obtained for indicating the relationship between the angle of rotation $\phi$ and the specific angle of rotation $\alpha$ :

$$
\phi=\frac{\alpha N M \pi}{1800 N_{A}}
$$

Furthermore, from comparison between equation (7) and equation (9), the following relationships can be obtained:

$$
\begin{gathered}
\varepsilon / \varepsilon_{0}=\varepsilon_{\infty}+S_{j} \\
\phi=\frac{N}{\lambda^{2}} g^{\prime} \\
g^{\prime} \equiv \frac{4 \pi^{2}}{3} c \beta\left(2+\varepsilon_{\infty}+S_{j}\right)=\frac{4 \pi^{2} c}{3} \beta\left(2+\varepsilon / \varepsilon_{0}\right)
\end{gathered}
$$

Equation (14) only includes the physical constants and some values unique to the material, and thus $g^{\prime}$ in equation (14) can be considered to be the constant value unique to sugared water. It should be noted that $g^{\prime}$ is independent from wavelength values, and thus, $\lambda$ instead of $\lambda_{0}$ is used in equation (13).

Further comparing equation (11) and equation (13), the following expression can be obtained:

$$
g^{\prime}=\frac{\alpha N M \pi}{1800 N_{A}} \cdot \frac{\lambda^{2}}{N}=\frac{\alpha M \pi \lambda^{2}}{1800 N_{A}}
$$

By respectively employing the following values: $\alpha=66.034625 ; M=342.2964 ; N_{A}=6.02 \times 10^{23}$; and $\lambda=589.29 \times 10^{-9}$, the value of $g$ ' can be calculated as follows:

$$
g^{\prime}=\frac{\alpha M \pi \lambda^{2}}{1800 N_{A}}=\frac{66.034625 \cdot 342.2964 \cdot \pi \cdot\left(589.29 \cdot 10^{-9}\right)^{2}}{1800 \cdot 6.02 \cdot 10^{23}}=2.276 \cdot 10^{-35}
$$


Further introducing the above value of $g^{\prime}$ into equation (13), the following expression can be derived:

$$
\phi=\frac{N}{\lambda^{2}} \cdot 2,276 \cdot 10^{-35}
$$

Equation (17) provides us with the value of the angle of rotation $\phi$ for sugared water with any wavelength values $\lambda$ of travelling light beams and any concentration values (actually the any values of $N$ which indicates the number of molecules in $1 \mathrm{~cm}^{3}$ ). In other words, the rotation angle values of sugared water at any concentration levels with any wavelength values of travelling light beams can be expected by employing equation (17). This kind of general expression for the angle of rotation $\phi$ of sugared water cannot be found in previous literatures.

By employing the similar procedures, like general expressions can be obtained for expressing the relationship between the angle of rotation $\phi$ and $\lambda, N$ for any other materials.

\section{COMPARISONS BETWEEN EXPECTED VALUES AND EXPERIMENTAL VALUES FOR THE ANGLE OF ROTATION IN SUGARED WATER}

For the purpose of obtaining verification of the general expression in equation (17), measurements of the angle of rotation with sugared water were done and the measured valued were compared with the corresponding predicted values to be calculated from equation (17).

More specifically, high-concentration sugared water was poured into a hand-made water tank with $9 \mathrm{~cm}$ in height, $9 \mathrm{~cm}$ in width and $32 \mathrm{~cm}$ in length, made of acrylic plates with $3 \mathrm{~mm}$ thick. The sugared water was prepared by allowing 700 $\mathrm{g}$ of sugar to be dissolved into $300 \mathrm{~g}$ of water while heating, resulting in about $73 \%$ of the Brix value. Although the Brix value can be calculated by dividing the amount $(\mathrm{g})$ of dissolved sugar by the sum of the amount $(\mathrm{g})$ of water and the amount $(\mathrm{g})$ of sugar, the Brix value in this investigation was actually measured with a saccharimeter.

A pair of polarizing plates were employed in a cross Nichol arrangement (i.e., perpendicular to each other) with the first plate placed at one end of the water tank and the second one put into the sugared water in the water tank. A laser source was placed at the outside of and adjacent to the end of the water tank where the first polarizing plate was placed. At the opposite end of the water tank, a solar panel was placed as a photo-detecting member. Figure 2 shows a photo of such an experimental setup. Both sides as well as a bottom of the water tank were covered with black paper so as to avoid any optical disturbances.

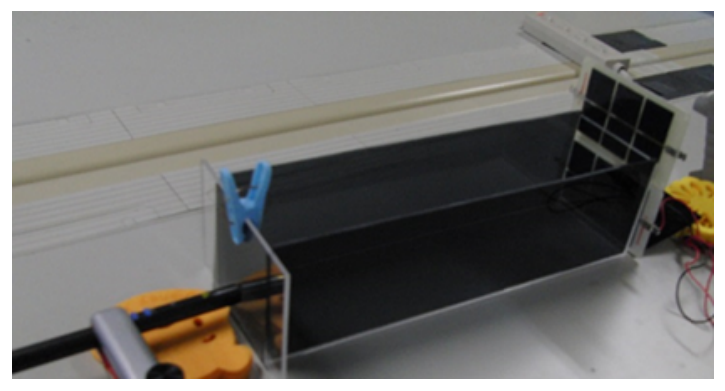

Figure 2. The experimental setup.

Laser beams from the laser source was allowed to be incident on and go through the first polarizing plate and then to enter the sugared water in the tank and travel along the longitudinal direction of the tank towards the solar panel placed at the opposite end. The second polarizing plate in the sugared water was moved from the initial position at the lasersource-side end of the tank (distance $z=0 \mathrm{~cm}$ between the two polarizing plates) to the opposite end of the water tank (distance $z=32 \mathrm{~cm}$ between the two polarizing plates) by $1 \mathrm{~cm}$. The laser beams were linearly polarized when entered into the sugared water, and then were subjected to optical rotation of the sugared water so that their polarization axis was caused to rotate. Due to the cross Nichol arrangement of the pair of polarizing plates, the intensity of the laser beams incident onto the solar panel at the opposite end of the water tank varied dependent on the position of the second polarizing plate (i.e., the distance $z$ between the two polarizing plates), and thus the output voltage level from the solar panel (in other words, the intensities of transmitted laser beams) was changed accordingly. 


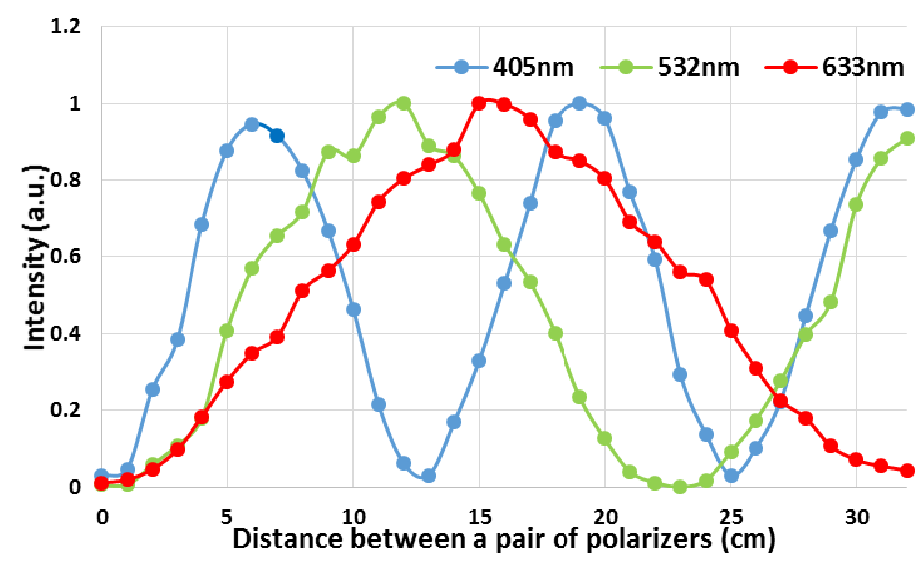

Figure 3. Measured changes in the intensity levels of transmitted laser beams.

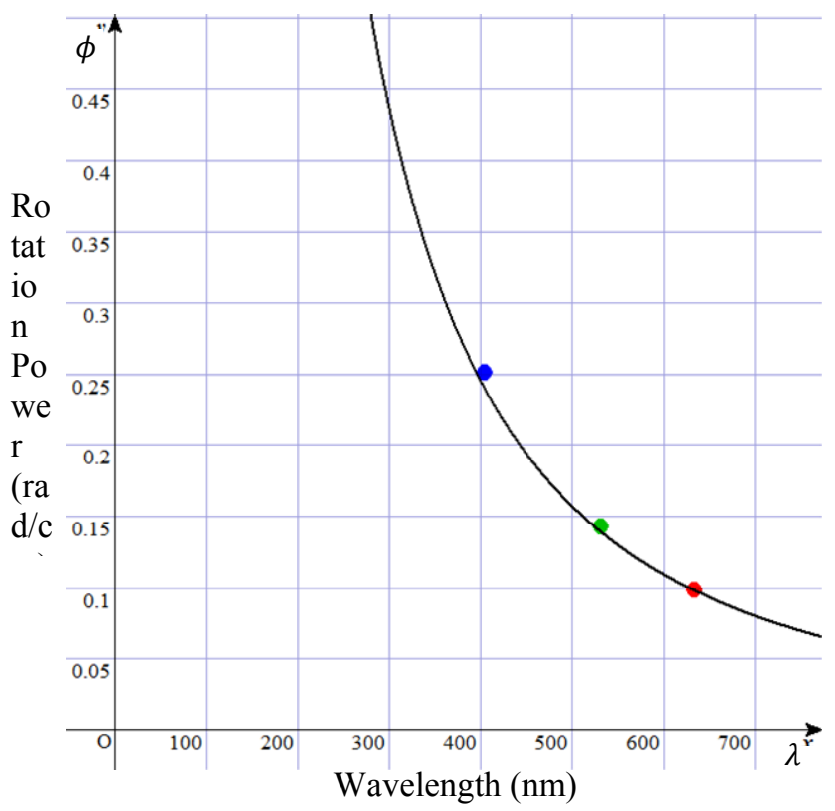

Figure 4. Predicted (solid line) and measured (plots) characteristics of the angle of rotation over wavelengths of transmitted laser beams with the angle of rotation $(\mathrm{rad} / \mathrm{cm})$ on the vertical axis and the wavelength $(\mathrm{nm})$ on the horizontal axis.

Figure 3 shows the measured changes in the intensities of transmitted laser beams with three different wavelengths (blue: $405 \mathrm{~nm}$, green: $532 \mathrm{~nm}$, and red: $633 \mathrm{~nm}$ ). The horizontal axis indicates the distance $z$ between the two polarizing plates (polarizers), and the vertical axis indicates the intensity levels normalized with respect to the maximum measured level for each wavelength.

As can be seen, the intensity levels of the transmitted laser beams showed sinusoidal-wave like changes over the distance between the two polarizing plates, and the interval in their changes became larger at the longer wavelengths. The similar measurements were also done at the different concentrations (at Brix values of $62 \%$ and $53 \%$ ), and the similar tendencies were observed (not shown here), while the interval in the changes in the intensities of transmitted laser beams became larger (i.e., taking a longer distance) at lower concentration levels of the sugared water.

From these measured data, the angle of rotation for each wavelength was calculated. In addition, as discussed previously, the angle of rotation for each wavelength was able to be predicted from the calculation of the following equation: 


$$
\begin{gathered}
\phi=\frac{N}{\lambda^{2}} \cdot 2,276 \cdot 10^{-35} \\
N=\frac{W \cdot N_{A}}{M \cdot V}
\end{gathered}
$$

where $V\left(\mathrm{~cm}^{3}\right)$ indicates the volume of the solution (sugared water), and $W(\mathrm{~g})$ indicates the mass of sugar dissolved into the solution. The prediction results of the angle of rotation, calculated with equation (17), were drawn in Figure 4, in which the measured values were also plotted. Relatively satisfactory matching can be recognized between the predictions and the results determined from the actual measurements. In other words, from the general equation shown as equation (17), the angle of rotation to be obtainable in sugared water successfully became predictable at any concentration levels with light beams at any wavelength.

\section{PREDICTIONS OF THE INTENSITIES OF TRANSMITTED LIGHT BEAMS}

As the next step, predictions of the intensity levels of transmitted light beams (the actual measured results for the laser sources were shown in Figure 3 were tried.

More specifically, when an optically active material is disposed between a pair of polarizing plates in a cross Nichol arrangement and the linearly polarized light beams are allowed to travel therein along the z axis, the electric field $\boldsymbol{E}_{\boldsymbol{0}}$ of the travelling polarized light beams can be expressed as follows:

$$
\boldsymbol{E}_{\boldsymbol{o}}=\left(\begin{array}{ll}
1 & 0 \\
0 & 0
\end{array}\right)\left(\begin{array}{cc}
\cos (\phi z) & \sin (\phi z) \\
-\sin (\phi z) & \cos (\phi z)
\end{array}\right)\left(\begin{array}{l}
0 \\
1
\end{array}\right)=\sin (\phi z)
$$

where $z$ indicates the position along the $\mathrm{z}$ axis, and $\phi$ indicates the angle of rotation of the optically active material such as sugared water. The value $\phi$ can be actually measured, or alternatively, can be predicted in accordance with the proposed equation as mentioned above.

The intensity $I$ of light beams can be expressed as $\boldsymbol{E}_{0}^{2}$, and therefore, expressed as follows:

$$
I=\sin ^{2}(\phi z)
$$

By inputting the predicted values of the angle of rotation into $\phi$ in equation (20), the intensity changes of the transmitted laser beams for the three wavelengths (i.e., blue: $405 \mathrm{~nm}$, green: $532 \mathrm{~nm}$, and red: $633 \mathrm{~nm}$ ) are predictable. The results are shown in Figure 5.

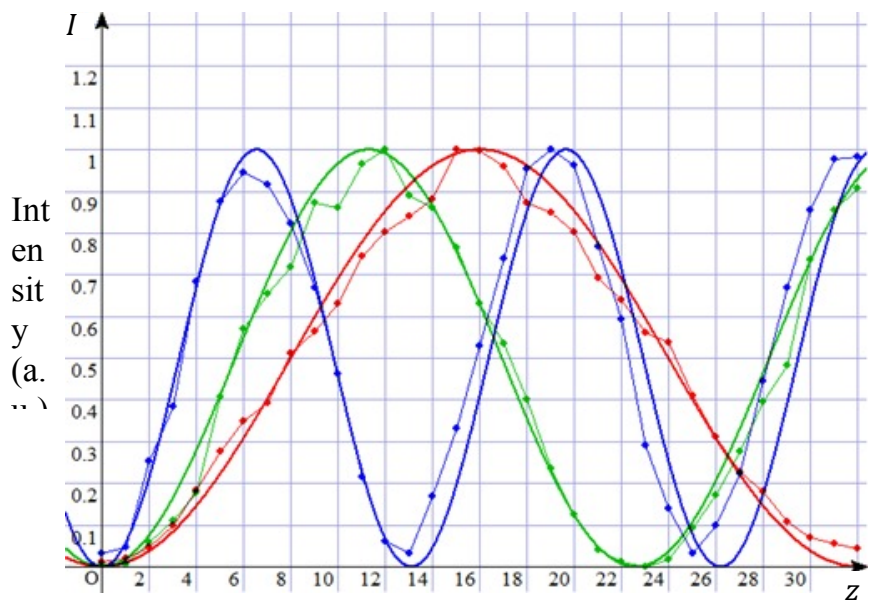

Distance between a pair of polarizing plates $(\mathrm{cm})$

Figure 5. Predicted (solid line) and measured (plots) characteristics of the intensity changes of the transmitted laser beams in the sugared water at the concentration of $73 \%$. 
In Figure 5, the horizontal axis indicates the distance between the pair of polarizing plated in the sugared water, and the vertical axis indicates the intensity of transmitted light beams with normalized with respect to the respective maximum levels. The solid lines show the predicted results, while the plots show the actually measured data as previously shown in Figure 3. Relatively satisfactory matching can be recognized between the predictions and the results obtained from the actual measurements. In other words, the intensity levels of the laser beams transmitted in sugared water successfully became predictable at any concentration levels with light beams at any wavelength.

As the further step, predictions of changes in visible colors are also become possible in the case where a white light (for example, an white-color LED torch including a red LED component, a green LED component, and a blue LED component to produce white-color light beams) is used as a light source. Figure 6 shows: (a) actually observed changes in visible colors of the transmitted light beams emitted from the white-color LED torch (indicated as the function of distances between a pair of polarizing plates in sugared water); and (b) corresponding predictions in changes in colors to be observed. Relatively satisfactory matching between the actual observation results in (a) and the predicted color changes in (b) were found.

By employing such expectations in colors observable in experimental demonstrations which employs optical rotation phenomena of sugared water, more effective educational programs can be designed.

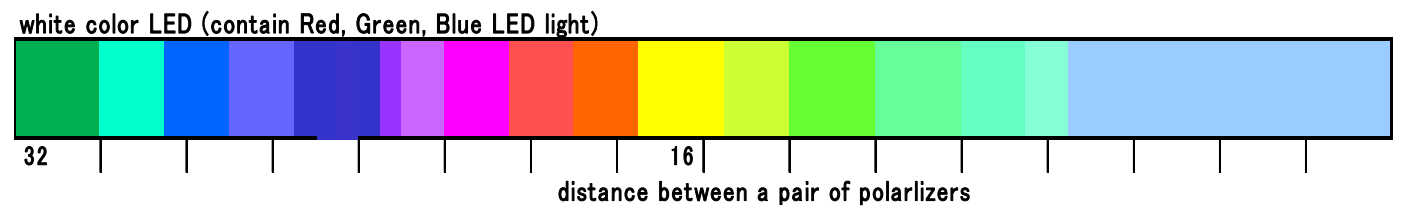

(a) actually observed changes in visible colors of the transmitted light beams emitted from the white-color LED torch

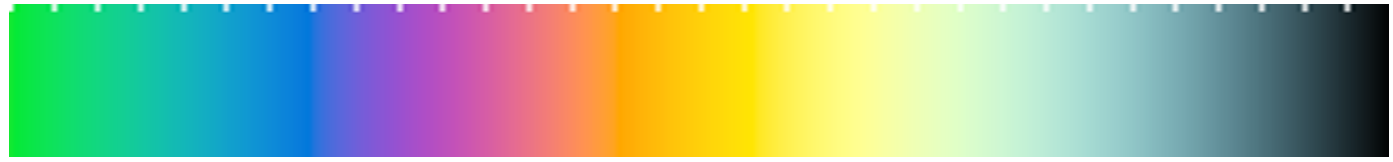

(b) corresponding predictions in changes in colors to be observed

Figure 6. Predictions of changes in visible colors for white-color light beams.

\section{CONCLUSIONS}

With respect to the coloring phenomena to be caused by optical rotation of polarized light beams in sugared water, an equation was successfully established to predict a theoretically expectable value for the angle of rotation for light beams of any colors (wavelengths) travelling in sugared water of any concentrations. The theoretically expected results obtained from the equation were then compared with the values determined from the experiments, and confirmed to show satisfactory matches. Furthermore, based on the theoretical results obtainable from the equation, the intensities of transmitted laser beams, as well as colors to be observable when a white-color LED torch was employed as a light source, were become predictable, and they were well-matched with the actual observation results.

\section{ACKNOWLEDGEMENT}

The authors are very grateful to Associate Professor Dr. Kenji Harada of Kitami Institute of Technology, Japan, for his useful discussions and suggestions.

\section{REFERENCES}

[1] Hecht, E., [Optics, 4th edition], Addison Wesley, San Francisco, 358-365 (2002).

[2] Huard, S., [Polarization of Light], John Wiley \& Sons, 64-65 (1997). 